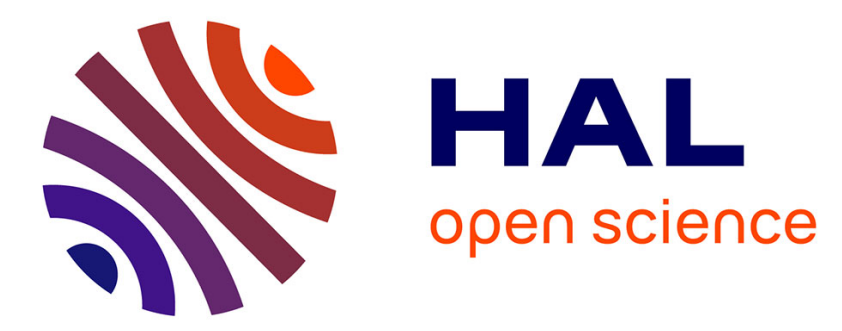

\title{
Delocalized hole transport coupled to sub-ns tryptophanyl deprotonation promotes photoreduction of class II photolyases
}

Fabien Lacombat, Agathe Espagne, Nadia Dozova, Pascal Plaza, Elisabeth Ignatz, Stephan Kiontke, Lars-Oliver Essen

\section{To cite this version:}

Fabien Lacombat, Agathe Espagne, Nadia Dozova, Pascal Plaza, Elisabeth Ignatz, et al.. Delocalized hole transport coupled to sub-ns tryptophanyl deprotonation promotes photoreduction of class II photolyases. Physical Chemistry Chemical Physics, 2018, 20 (39), pp.25446 - 25457. 10.1039/c8cp04548h . hal-01913266

\section{HAL Id: hal-01913266 https://hal.science/hal-01913266}

Submitted on 24 Jan 2019

HAL is a multi-disciplinary open access archive for the deposit and dissemination of scientific research documents, whether they are published or not. The documents may come from teaching and research institutions in France or abroad, or from public or private research centers.
L'archive ouverte pluridisciplinaire HAL, est destinée au dépôt et à la diffusion de documents scientifiques de niveau recherche, publiés ou non, émanant des établissements d'enseignement et de recherche français ou étrangers, des laboratoires publics ou privés. 


\title{
Delocalized Hole Transport Coupled to Sub-ns Tryptophanyl Deprotonation Promotes Photoreduction of Class II Photolyases
}

Fabien Lacombat, ${ }^{a}$ Agathe Espagne, ${ }^{a}$ Nadia Dozova, ${ }^{a}$ Pascal Plaza, ${ }^{* a}$ Elisabeth Ignatz, ${ }^{b}$ Stephan Kiontke, ${ }^{b}$ Lars-Oliver Essen ${ }^{*}{ }^{b}$

${ }^{a}$ PASTEUR, Département de chimie, École normale supérieure, PSL University, Sorbonne Université, CNRS, 75005 Paris, France. E-mail: pascal.plaza@ens.fr

${ }^{b}$ Department of Chemistry, LOEWE Center for Synthetic Microbiology, Philipps University, 35032 Marburg, Germany. E-mail: essen@chemie.uni-marburg.de

KEYWORDS: class II photolyase, photoactivation, photoreduction, electron transfer, flavin, tryptophan, deprotonation, femtosecond, ultrafast, transient absorption spectroscopy, anisotropy

\begin{abstract}
Class II photolyases utilize for the photoreduction of their flavin cofactor (FAD) a completely different tryptophan triad than most other photolyases and cryptochromes. To counter sped-up back electron transfer, they evolved an unusually fast deprotonation of the distal tryptophanyl radical cation $\left(\mathrm{WH}^{\bullet+}\right)$ that is produced after excitation of the flavin. We studied the
\end{abstract}


primary aspects of oxidized FAD photoreduction by ultrafast transient absorption spectroscopy, using the class II photolyase from Methanosarcina mazei. With a time constant of $9.2 \mathrm{ps,} \mathrm{the}$ initial reduction step of the excited flavin by the proximal $\mathrm{W}_{381}$ tryptophan proceeds almost twentyfold slower than in other photolyases carrying oxidized FAD, most likely because of the larger distance between the flavin and the proximal tryptophan. The thus formed $\mathrm{W}_{381} \mathrm{H}^{\bullet+}$ radical is tracked by transient anisotropy measurements to migrate in 29 ps with delocalization over several members of the tryptophan triad. This 29 -ps phase also includes the decay of a small fraction of excited flavin, reacting on a slower timescale, and a partial recombination of the $\mathrm{FAD}^{\bullet-} / \mathrm{WH}^{\bullet+}$ radical pair. A final kinetic phase in $230 \mathrm{ps}$ is assigned to the deprotonation of $\mathrm{W}_{388} \mathrm{H}^{\bullet+}$ that occurs in competition with partial charge recombination. Interestingly, we show by comparison with the Y345F mutant that this last phase additionally involves oxidation of the $\mathrm{Y}_{345}$ phenolic group by $\mathrm{W}_{388} \mathrm{H}^{\bullet+}$, producing a small amount of neutral tyrosyl radical $\left(\mathrm{YO}^{\bullet}\right)$. The rate of this electron transfer step is about six orders of magnitude faster than the corresponding oxidation of $\mathrm{Y}_{345}$ by the deprotonated $\mathrm{W}_{388}{ }^{\bullet}$ radical. Unlike conventional photolyases, where the electron hole accumulates on the distal tryptophan before the much slower tryptophanyl deprotonation, our data show that delocalized hole transport is concomitantly concluded by ultrafast deprotonation of $\mathrm{W}_{388} \mathrm{H}^{\bullet+}$.

\section{MANUSCRIPT TEXT}

\section{INTRODUCTION}

Photolyases are flavoproteins able to photorepair DNA damaged by UV irradiation, presenting pyrimidine dimers. ${ }^{1-5}$ Depending on their specificity for the repair of cis,syn-cyclobutane 
pyrimidine dimers (CPD) and pyrimidine(6-4)pyrimidone photoproducts, photolyases are divided into CPD and (6-4) photolyases, respectively. ${ }^{6}$ Together with the blue-light sensing cryptochromes these photolyases form a large and highly diverse superfamily of flavoenzymes, which occur almost ubiquitously among sunlight exposed organisms from all kingdoms of Life. ${ }^{\dagger}$ In particular, CPD photolyases are subdivided into three classes: class I photolyases are the most well-known and occur in microbes, class II photolyases are predominantly found in higher, multicellular eukaryotes and class III photolyases occur in some eubacteria. Class II photolyases are particularly interesting as they exhibit significant deviations in terms of sequence and structure of the photocatalytic site, ${ }^{7,11-14}$ as well as of their photorepair mechanism. ${ }^{15}$ All photolyases non-covalently bind a chromophoric cofactor, flavin adenine dinucleotide (FAD), which is essential for the DNA repair process and adopts a unique U-shaped conformation. ${ }^{1,4,5}$ In brief, $\mathrm{FAD}$ in its fully reduced form $\left(\mathrm{FADH}^{-}\right)$absorbs blue/UV-A light and subsequently transfers an electron to the DNA lesion, which elicits a series of reactions leading to the restoration of intact pyrimidine nucleobases and a final return of the electron to the transiently oxidized flavin. If the flavin cofactor is instead initially oxidized $\left(\mathrm{FAD}_{\mathrm{ox}}\right)$ or semi-reduced $\left(\mathrm{FADH}^{\circ}\right)$, photolyases have the additional ability to restore the active $\mathrm{FADH}^{-}$form by a reaction called photoactivation. ${ }^{1,4,5}$ During photoactivation, the oxidized or semi-reduced flavin is excited and subsequently abstracts an electron from a nearby electron donor. The oxidation hole then migrates towards the surface of the photolyase, where it is reduced by an external electron donor, thereby stabilizing the reduced flavin. This photoreduction process is also found in the closely related cryptochromes, which are blue-light receptors responsible for different biological

\footnotetext{
$\dagger$ The evolutionary links between the different members of the photolyase and cryptochrome superfamily (PCSf) may be found in various reports. ${ }^{7-10}$
} 
photoinduced responses, and plays here the role of a trigger of the phototransduction process. ${ }^{1,16,17}$ Ultrafast spectroscopy has been extensively used to study the primary steps of the photoreduction of class I photolyases, ${ }^{18-23}(6-4)$ photolyases $^{24-27}$ as well as different types of cryptochromes. ${ }^{24,25,28}$ These studies have shown that when the oxidized flavin is excited, it is extremely rapidly reduced, in less than $1 \mathrm{ps}$, by a nearby tryptophan residue to produce an $\mathrm{FAD}^{\bullet}$ $/ \mathrm{WH}^{\bullet+}$ radical pair. ${ }^{\star}$ Slower steps observed in the ps to sub-ns regime have been assigned to the migration of the oxidation hole along a chain of three conserved tryptophan residues, occurring in competition with partial charge recombination of the radical pair. In some cases, this tryptophan triad serving as electron transfer chain is extended by an additional tryptophan ${ }^{27,29}$ or tyrosine residue. ${ }^{30-32}$ When starting with the semi-reduced flavin, the onset of photoreduction is significantly slower, in about $30 \mathrm{ps}$, and may become the rate limiting step of the overall charge separation process. ${ }^{4}$ Deprotonation of the distal $\mathrm{WH}^{\bullet+}$ radical to the neutral $\mathrm{W}^{\bullet}$ species then follows on a much longer timescale, e.g. $200 \mathrm{~ns}$ for the class I photolyase of $E$. coli $^{18,19}$ ) or 2.5 $\mu$ s for the (6-4) photolyase of Xenopus laevis. ${ }^{26}$

The photoactivation of class II photolyases is less well documented. One hallmark of class II photolyases is their dependence on a completely different tryptophan triad than the classical one found in most photolyases and cryptochromes. ${ }^{12,13}$ Figure 1 illustrates this triad $\left(\mathrm{W}_{381}, \mathrm{~W}_{360}\right.$ and $\mathrm{W}_{388}$ ) for the class II photolyase of Methanosarcina mazei (noted MmCPDII), according to the crystal structure of Kiontke et al. ${ }^{13}$ (PDB entry 2XRY). Recently, Müller et al..$^{33}$ have shown by ns-ms transient absorption spectroscopy that the distal $\mathrm{W}_{388} \mathrm{H}^{\bullet+}$ radical produced after irradiation

\footnotetext{
\$ The $\mathrm{WH}^{\bullet+}$ notation emphasizes that the protonated character of this tryptophanyl radical. The deprotonated tryptophanyl is noted $\mathrm{W}^{\bullet}$. Similarly, $\mathrm{YO}^{\bullet}$ indicates a neutral, deprotonated tyrosyl radical.
} 
of the flavin deprotonates about three order of magnitude faster than in any class I photolyase, namely in $350 \mathrm{ps}$. Here, a protein-bound cluster of water molecules was suggested to serve as the primary proton acceptor of this reaction. This study additionally showed that a conserved tyrosine residue situated at very short distance of $\mathrm{W}_{388}\left(\mathrm{Y}_{345}\right.$, see Figure 1), also participates in the photoactivation process. Accordingly, it was proposed that $Y_{345}$ gets partially oxidized in the sub-ms regime, in competition with recombination of the $\mathrm{FAD}^{\bullet} / \mathrm{W}_{388^{\circ}}$ pair. The formed $\mathrm{FAD}^{\bullet}$ $/ \mathrm{Y}_{345} \mathrm{O}^{\bullet}$ pair finally recombines in $1.1 \mathrm{~ms}$.

We report here the first ultrafast spectroscopic study of the photoactivation of a class II photolyase, namely $\mathrm{MmCPDII}$. The major objective is to explore the dynamics of this reaction in the fs-ps timescale that escaped Müller et al.'s study, ${ }^{33}$ and compare it to other known photolyases and cryptochromes. The present study has been conducted on both the wild-type (WT) of $M m \mathrm{CPDII}$ and its Y345F mutant (shortcuts WT and Y345F will be subsequently used to designate them. Y345F cancels the electron donating character of $\mathrm{Y}_{345},{ }^{33}$ which allows to evaluate the possible role of $\mathrm{Y}_{345}$ in the short time scale. Notably, the $\mathrm{Y} 345 \mathrm{~F}$ mutation was shown by X-ray crystallography to cause no substantial disturbance of the crystal structure. ${ }^{14}$

Furthermore, distinguishing between putative $\mathrm{WH}^{\bullet+}$ intermediates as expected to be transiently formed within the tryptophan triad during the charge migration process is not straightforward because they have the same chemical nature and hence similar spectroscopic properties. As in several previous studies, ${ }^{19,22,25,27,28,34}$ polarized transient absorption spectroscopy was used here to access the orientations of the involved species through the associated transient anisotropy. Standard measurement of the population kinetics and isotropic spectra is granted by the reconstructed signal at the magic angle. 


\section{MATERIALS AND METHODS}

\subsection{Sample preparation}

The Methanosarcina mazei class II photolyase (MmCPDII) was prepared as previously described. ${ }^{7,13,33}$ For the transient absorption experiments, we used a $10 \mathrm{mM}$ Tris buffer at $\mathrm{pH} 8.0$ with $100 \mathrm{mM} \mathrm{NaCl}$ and $10 \%$ glycerol. The $\mathrm{D}_{2} \mathrm{O}$ buffer was composed accordingly with heavy water (99.9 atom \% D, Sigma-Aldrich) but contained standard glycerol, which yields a residual amount of labile protons of the order of $4 \%$. Some samples were concentrated with centrifugal concentrators (Vivaspin 500, Sartorius or Amicon Ultra-4, Sigma-Aldrich; cutoff: $30 \mathrm{kDa}$ ) to achieve suitable optical densities. Immediately before the experiments, the protein solutions were passed through a gel-filtration column (Micro Bio-Spin6, BioRad), equilibrated with the working buffer, to eliminate unbound FAD. Sample volumes of $\sim 50 \mu \mathrm{L}$ of concentrated solution were used for the experiments, which were all performed at $10^{\circ} \mathrm{C}$.

\subsection{Steady-state spectroscopy}

Steady-state absorption spectra were measured with a Cary 300 (Varian) double-beam UV/Vis spectrophotometer. The steady-state absorption spectra of the MmCPDII samples used for transient absorption spectroscopy are shown in $\mathrm{ESI}^{\S}-\S 1$ (Figure $\mathrm{S} 1$ ). They essentially exhibit the characteristic bands of $\mathrm{FAD}_{\mathrm{ox}}$ at 377 and $444 \mathrm{~nm}$. Taking the commonly used value of the absorption coefficient of $\mathrm{FAD}_{\mathrm{ox}}$ at the $444-\mathrm{nm}$ maximum, ${ }^{35}$ the concentration of $\mathrm{FAD}_{\mathrm{ox}}$ is

\footnotetext{
$\S$ Electronic Supplementary Information
} 
estimated to $\sim 145 \mu \mathrm{M}$ in the case of WT in $\mathrm{H}_{2} \mathrm{O}$ buffer, $109 \mu \mathrm{M}$ for WT in $\mathrm{D}_{2} \mathrm{O}$ and $278 \mu \mathrm{M}$ for Y345F in $\mathrm{H}_{2} \mathrm{O}$ (see ESI-§1).

\subsection{Femtosecond transient absorption spectroscopy}

Polarized broadband transient absorption spectra were recorded by the pump-probe technique, with white-light continuum probe, as previously described. ${ }^{25,27}$ The continuum probe was generated by focusing a few $\mu \mathrm{J} /$ pulse of the $775-\mathrm{nm}$ beam on a moving $\mathrm{CaF}_{2}$ plate. The pump beam at $475 \mathrm{~nm}(55 \mathrm{fs})$ was generated by using a two-stage noncollinear optical parametric amplifier (NOPA, Clark MXR). The energy used to excite the samples was set to about $150 \mathrm{~nJ}$ per pulse, focused on a surface of about $15000 \mu \mathrm{m}^{2}$ (at half of maximum of intensity). The actual fraction of excited molecules in the probed volume was estimated to be about $3 \%$ by comparison with a reference sample of tris-(2,2'-bipyridyl)-ruthenium(II) complex,${ }^{36}$ which lies in the linear regime. Systematic measurements on the $\left[\mathrm{Ru}(\mathrm{bpy})_{3}\right]^{2+}$ complex were used to ensure (i) that no major alteration of the pump-probe spatial overlap occurred upon varying the pump-probe delay over the 3.2-ns range of an optical delay line, ${ }^{37}$ and (ii) that experiments recorded on different days were comparable. These measurements also allowed to verify that the reconstructed anisotropy of our reference compound decays to zero at long delay times, due to rotational diffusion, as expected from a correct measurement of the parallel and perpendicular data.

During all experiments the $\mathrm{MmCPDII}$ samples were kept at $10^{\circ} \mathrm{C}$ with the help of a circulating thermostated bath. Steady-state absorption spectra of the samples were regularly recorded during the experiments to check that no substantial degradation occurred.

The polarized differential absorption spectra $\left(\Delta \mathrm{A}_{\|}\right.$and $\left.\Delta \mathrm{A}_{\perp}\right)$, obtained from the transmitted probe beam intensities, were first corrected from the chirp of the probe beam, which was independently 
measured by recording cross-phase modulation ${ }^{38}$ (XPM) in the pure solvent. These raw polarized spectra are not shown. Corrected $\Delta \mathrm{A}_{\|}$and $\Delta \mathrm{A}_{\perp}$ spectra were then replaced by isotropic transient absorption spectra $\left(\Delta \mathrm{A}_{\text {iso }}\right)$ and transient anisotropy spectra $(\mathrm{r})$ as calculated from the standard definitions of Equations 1 and 2, which can be univocally inverted to retrieve the polarized data. Scattering of the pump beam was masked in the spectra, both for data analysis and figure presentation.

$$
\left\{\begin{array}{l}
\Delta \mathrm{A}_{\text {iso }}=\frac{1}{3}\left(\Delta \mathrm{A}_{\|}+2 \Delta \mathrm{A}_{\perp}\right) \\
\mathrm{r}=\frac{\Delta \mathrm{A}_{\|}-\Delta \mathrm{A}_{\perp}}{\Delta \mathrm{A}_{\|}+2 \Delta \mathrm{A}_{\perp}}
\end{array}\right.
$$

Note that the isotropic spectra are free from rotational diffusion and intrinsic polarization effects. They describe the pure population dynamics and allow for a direct comparison with steady-state spectra of known species. The anisotropy dynamics presented below reflect intrinsic changes within the protein since rotational diffusion of $\mathrm{MmCPDII}$ as a whole (molecular mass: 55,832 $\mathrm{Da}$ ) is not expected to be active during the time window of our measurements (3.2 ns). It has indeed been shown for similar photolyases that it takes place in the timescale of tens of ns. ${ }^{27,34}$ The intrinsic dynamics of the protein being characterized by large population changes (electron and proton transfers, charge recombination; see Results and Discussion), we assumed that minor contributions to the anisotropy due to hindered movements of the various molecular species involved in the dynamics (FAD, tryptophans) could be neglected. Consequently, the anisotropy and isotropic signal were considered to follow the same dynamics. The success of our simultaneous fit of the parallel and perpendicular data with identical exponential functions (see §2.4) justifies our approximation.

\subsection{Kinetic analysis}


The transient absorption data were globally fitted ${ }^{39}$ to a sum of exponential functions, convoluted by a Gaussian function representing the instrument response function (IRF; found to be around 180 fs FWHM). Singular value decomposition ${ }^{40}$ was applied to the data in order to ease the fitting procedure and to filter out noise; care was taken to keep enough singular values (15) to ensure no information loss. The full data set was analyzed and the XPM artifact ${ }^{38}$ occurring during pump-probe overlap was empirically accounted for by a sum of the IRF Gaussian and its

first four time derivatives. ${ }^{41}$ The fits were made simultaneously on the parallel and perpendicular data, sharing the same sum of exponentials. The effective fits of the isotropic or anisotropy data presented below are deduced from this root fit, using Equations 1 and 2.

Spectra of pre-exponential factors were obtained from the fit, for each polarization, but are not presented here. We use instead the evolution-associated difference spectra (EADS), calculated from them. ${ }^{39}$ They correspond to a virtual cascading model with $100 \%$ quantum yield between one state and the following. EADS are used as a convenient tool to summarize the spectral dynamics but are not meant to represent any actual kinetic model. The polarized EADS deduced from the fit were converted into isotropic EADS and evolution-associated anisotropy spectra (EAAS) by using Equations 1 and 2. Note that EADS1 is the differential spectrum extrapolated at $\mathrm{t}=0$, deconvoluted from IRF and separated from XPM.

\subsection{Spectral analysis}

In the present work, we tried to quantitatively analyze the transient absorption spectra, more specifically the isotropic EADS, in terms of a sum of positive and negative contributions. Positive terms come from the absorption of different photoproducts $\left(\mathrm{FAD}^{\bullet}, \mathrm{WH}^{\bullet+}, \mathrm{W}^{\bullet}\right)$, while a 
negative sign is attached to the $\mathrm{FAD}_{\mathrm{ox}}$ bleaching. The goal was to ensure that the qualitative interpretations deduced by inspection of the spectra (§4.1) are correct.

A number of reference spectra were taken from literature, and are gathered in ESI-§4.1 (Figure S7). A simple linear combination of these however yields poor approximations of the experimental spectra, as previously observed for other photolyases. ${ }^{25,27}$ This is due to the fact that the spectra of the different transient species may change according to their local environment, especially when going from solution to the photolyase context. To overcome this difficulty, we modified each reference spectrum with the help of two empirical parameters: a shift (s) and a scaling factor (k). In that way, spectrum $\varepsilon(\lambda)$ becomes $\varepsilon^{\prime}(\lambda)=\varepsilon(\mathrm{k}(\lambda-\mathrm{s}))$. The chosen procedure was then to fit the EADS (between 350 and $700 \mathrm{~nm}$ ) with a weighted sum of the modified reference spectra, the $\mathrm{s}$ and $\mathrm{k}$ factors being adjustable parameters. The amplitudes should in principle represent concentrations of each species and be linked by conservation relations (between bleaching contribution and sum of products, negative and positive charge, etc). However, we dropped these conditions to allow the absorption coefficient of each species to vary independently with respect to the reference spectra.

The limit of this approach is that if too many species with overlapping spectra are involved, good fits may be obtained but with questionable value (see §4.2.1). We thus limited ourselves to cases where the number of species in the sum could be kept low and their spectral overlap does not generate ambiguities. In particular, we focused on the pairwise difference between consecutive EADS. The idea is here to fit the $\mathrm{EADS}_{\mathrm{i}+1}-\phi \mathrm{EADS}_{\mathrm{i}}$ difference, $\phi$ being the yield of the $\mathrm{i} \rightarrow \mathrm{i}+1$ reaction. This approach allows to exclude the fraction $(1-\phi)$ of the transient population at step i that does not further react but rather returns to the initial configuration, i.e. by charge 
recombination. In that way only changes happening in the reactive fraction of the transient population are included. At a practical level, EADS ${ }_{i+1}$ is fitted with EADS $_{\mathrm{i}}$ as a fit component, multiplied by an adjustable factor $(\phi)$, plus additional components (see ESI- $\S 4)$. Note that this approach differs from fitting DADS $\mathrm{i}$, which includes charge recombination.

\section{RESULTS}

\subsection{Isotropic transient absorption spectroscopy}

\subsubsection{Spectral dynamics}

Figure 2 (A-C) presents isotropic transient absorption spectra of WT-MmCPDII, recorded between $200 \mathrm{fs}$ and $3.2 \mathrm{~ns}$ after excitation at $475 \mathrm{~nm}$. At a pump-probe delay of $200 \mathrm{fs}$ (black line in Figure 2A), the earliest time point without XPM artifact, the spectrum exhibits two negative bands: one dominated by the bleaching of the ground-state absorption (GSB) at $443 \mathrm{~nm}$, and the other by stimulated emission (SE) at $534 \mathrm{~nm}$. Three positive transient absorption bands are also observed: an intense and narrow peak at $366 \mathrm{~nm}$ (called $\left.\mathrm{TA}_{1}\right)$ with a shoulder at around $393 \mathrm{~nm}$, a small maximum at $507 \mathrm{~nm}\left(\mathrm{TA}_{2}\right)$ and a broad band over $616 \mathrm{~nm}\left(\mathrm{TA}_{3}\right)$. These are characteristic features of excited $\mathrm{FAD}_{\mathrm{ox}}\left(\mathrm{FAD}_{\mathrm{ox}}{ }^{*}\right)$, already observed in other photolyases or

cryptochrome, ${ }^{25,27,28}$ different flavoproteins, ${ }^{42,43}$ as well as for free $\mathrm{FAD}_{\text {ox }}$ in solution. ${ }^{41,44}$

The evolution of the transient absorption spectra may be divided into three phases, as follows:

- From 0.2 to $\sim 30$ ps (Figure $2 \mathrm{~A}$ ), the SE band decays and is substituted by a broad positive band, peaking at $598 \mathrm{~nm}$ and joining with the initial $\mathrm{TA}_{2}$ band through a local minimum at $533 \mathrm{~nm}$. This process indicates a loss of excited state. Meanwhile, a temporary isosbestic point is 
observed at $641 \mathrm{~nm}$ as the $\mathrm{TA}_{3}$ band decays. The $\mathrm{TA}_{1}$ band also undergoes significant changes, with a partial decay accompanied by the formation of a new secondary maximum at $404 \mathrm{~nm}$. Partial decay of the GSB band is also seen.

- Between $\sim 30$ and $\sim 160$ ps (Figure $2 B$ ), all bands decay partially while the local minimum at $533 \mathrm{~nm}$ disappears and leaves a very broad and flat positive band extending from $\sim 500$ to $\sim 650 \mathrm{~nm}$.

- Between $\sim 160$ and 3200 ps (Figure 2C), one observes a strong decay of the transient absorption around $600 \mathrm{~nm}$ while it does not change much around $500 \mathrm{~nm}$. The $\mathrm{TA}_{1}$ and GSB bands concomitantly decay partially. A remaining signal persists still at the end of our time window (3.2 ns).

The transient absorption spectra of WT-MmCPDII in a deuterated buffer were also measured and are provided in ESI-\$2.2 (Figure S3 A-C).They are essentially identical to those in $\mathrm{H}_{2} \mathrm{O}$ buffer, except for the third phase, which is distinctively slower. The transient absorption spectra of Y345F (Figure 2, D-F) are at first sight indistinguishable from those of WT (except for a better signal/noise ratio). We will go back to this issue in $\S 4.3$.

\subsubsection{Kinetic analysis}

The parallel and perpendicular transient absorption spectra of MmCPDII could be simultaneously and globally fitted with a sum of three exponential components followed by a plateau. Table 1 gathers the time constants obtained for all studied systems ( $2 \sigma$ errors, corresponding to a confidence interval of $95 \%$, are indicated for reference). An illustration of this fit may be found in ESI-§3.1 (Figure S4), with kinetic traces of both isotropic transient absorption and transient 
anisotropy of WT in $\mathrm{H}_{2} \mathrm{O}$, at selected wavelengths. Similar qualities of fit were obtained for the other two systems under study (not shown).

Figure $3(\mathrm{~A}, \mathrm{C})$ summarizes the evolution of the transient absorption spectra in terms of the isotropic EADS (see §2.4), for WT and $\mathrm{Y} 345 \mathrm{~F}$ in $\mathrm{H}_{2} \mathrm{O}$ buffer (those of WT in $\mathrm{D}_{2} \mathrm{O}$ may be found in ESI-§3, Figure S6A). The evolution phases sketched in $\$ 3.1 .1$ are here clearly quantified. The initial excited-state decay (EADS1 to EADS2) occurs in 9.2 ps for WT and $\mathrm{Y} 345 \mathrm{~F}$ in $\mathrm{H}_{2} \mathrm{O}$. This time constant slightly increases to $10.7 \mathrm{ps}$ for $\mathrm{WT}$ in $\mathrm{D}_{2} \mathrm{O}$. This difference is 1.5 times larger than the corresponding error (square root of sum of squares of individual errors) and may thus be considered as significant. The intermediate regime (EADS2 to EADS3) is characterized by time constants of 29 ps for WT in $\mathrm{H}_{2} \mathrm{O}, 50$ ps in $\mathrm{D}_{2} \mathrm{O}$ and 38 ps for $\mathrm{Y} 345 \mathrm{~F}$ in $\mathrm{H}_{2} \mathrm{O}$. All deviations

from WT in $\mathrm{H}_{2} \mathrm{O}$ observed here are significant as they are larger than the corresponding errors by a factor of about 2. The final step (EADS3 to EADS4) occurs in $230 \mathrm{ps}$ for $\mathrm{WT}_{\text {in }} \mathrm{H}_{2} \mathrm{O}, 570 \mathrm{ps}$ in $\mathrm{D}_{2} \mathrm{O}$ and 330 ps for $\mathrm{Y} 345 \mathrm{~F}$ in $\mathrm{H}_{2} \mathrm{O}$. Again, the differences with $\mathrm{WT}$ in $\mathrm{H}_{2} \mathrm{O}$ are significant, i.e. larger than the experimental errors by a factor of $\sim 2$. Figure S5 (ESI-§3.1) specifically illustrates the slowing-down of the slow decay kinetics of WT upon buffer deuteration.

\subsection{Time-resolved anisotropy}

The transient anisotropy spectra, calculated from the polarized data with Equation 2, are provided for reference in ESI- $\$ 2$ (Figure S2 for WT and Y345F in $\mathrm{H}_{2} \mathrm{O}$ buffer and Figure S3 (DF) for WT in $\mathrm{D}_{2} \mathrm{O}$ buffer). The evolution-associated anisotropy spectra (EAAS) coming for the global analysis procedure are shown in Figure 3 (B, D) for WT and Y345F in $\mathrm{H}_{2} \mathrm{O}$ buffer (and in Figure S6B for WT in $\mathrm{D}_{2} \mathrm{O}$ buffer). In brief, the three kinetic phases described above can also be clearly distinguished in anisotropy. The initial phase is characterized by very large changes 
between 500 and $650 \mathrm{~nm}$, due to the decay of SE (loss of $\mathrm{FAD}_{\text {ox }}$ excited state). Curiously, the anisotropy spectrum at the end of this phase (EAAS2) shows a dip around $535 \mathrm{~nm}$, evoking a similar feature in the corresponding isotropic spectrum (EADS2). This dip disappears during the second kinetic step (EAAS2 to EAAS3). We will go back in detail to this phenomenon in the Discussion section (§4.4.1). The last kinetic phase is accompanied by a substantial anisotropy decrease over $500 \mathrm{~nm}$. The final anisotropy becomes increasingly noisy above $\sim 650 \mathrm{~nm}$ simply because the corresponding isotropic spectrum (i.e. denominator of Equation 2) has only residual amplitudes at long wavelengths.

\section{DISCUSSION}

\subsection{Nature of the photoproducts}

In this section, we use the isotropic EADS to qualitatively identify the transient species produced upon excitation of $\mathrm{FAD}_{\mathrm{ox}}$ and decay of the excited state. Note that with the same arguments used to analyze the raw spectrum at $200 \mathrm{fs}$ in $\S 3.1$, it is clear that EADS1 is the differential spectrum of $\mathrm{FAD}_{\mathrm{ox}}$.

The first photoproduct (EADS2) is characterized by an altered $\mathrm{TA}_{1}$ band, with two local maxima (368 and $404 \mathrm{~nm}$ ), one single bleaching band and a broad absorption band around $600 \mathrm{~nm}$. This particular shape strongly evokes the initial photoproduct spectrum recorded with other oxidized photolyases ${ }^{25,27}$ and attributed to the $\mathrm{FAD}^{\bullet-} / \mathrm{WH}^{\bullet+}$ radical pair. $\mathrm{FAD}^{{ }^{-}}$is the reduced flavin obtained by electron transfer from a nearby tryptophan residue, thereby oxidized to $\mathrm{WH}^{\bullet+}$. In the present case, the $\mathrm{TA}_{1}$ maximum at $404 \mathrm{~nm}$ is a clear signature of a distinctive secondary 
absorption maximum of $\mathrm{FAD}^{--}$(see examples in glucose oxidase ${ }^{45}$, Drosophila cryptochrome, ${ }^{46}$ Anopheles gambiae cryptochrome $1^{47}$ ). The broad band around $600 \mathrm{~nm}$ is characteristic of $\mathrm{WH}^{\bullet+}$ in photolyases ${ }^{25,27}$ and is significantly red-shifted compared to the absorption spectrum of this species in solution. ${ }^{48}$ It is striking that the primary reduction of the $\mathrm{FAD}_{\mathrm{ox}} *$ occurs much more slowly than for other photolyases or cryptochromes, ${ }^{21,22,24,25,28}$ for instance by a factor of 19 compared to the (6-4) photolyase of Xenopus laevis $(X l 64) .{ }^{27}$ This is likely related to the greater distance between FAD and the proximal $\mathrm{W}_{381}$, namely $5.3 \AA$ in $\mathrm{MmCPDII}^{7}$ (Figure 1) vs. $4.0 \AA$ in $X l 64 .{ }^{27}$ The Moser-Dutton empirical formula for Marcus electron transfer ${ }^{49}$ indeed predicts a decrease of the rate constant by a factor of $\sim 6$ if distance is increased by $1.3 \AA$. The remaining factor of 3 might be sought in additional variations of the driving force $(\Delta \mathrm{G})$ and reorganization energy $(\lambda)$. It should be additionally noted that the dip of EADS2 at $533 \mathrm{~nm}$ reminds of the SE band of $\mathrm{FAD}_{\mathrm{ox}}{ }^{*}$ and suggests that a small fraction of $\mathrm{FAD}_{\mathrm{ox}}{ }^{*}$ might remain after the first decay phase. We will explore this hypothesis in a more quantitative manner in the following section.

EADS3 bears the same characteristics as EADS2 and may also be assigned to the $\mathrm{FAD}^{\bullet} / \mathrm{WH}^{\bullet+}$ pair. However, its intensity is only half of that of EADS2, which can be interpreted partial charge recombination of the radical pair, taking place during the second phase (29 ps for WT in $\mathrm{H}_{2} \mathrm{O}$ buffer). Furthermore, the 533-nm dip of EADS2 has disappeared in EADS3, which tentatively suggests that the above-hypothesized remaining fraction of $\mathrm{FAD}_{\mathrm{ox}}{ }^{*}$ has here completely decayed.

EADS4 is characterized by a massive loss of the $600-\mathrm{nm}$ band. Meanwhile the $\mathrm{TA}_{1}$ and GSB bands only show a moderate decay and no change of shape. In good agreement with the results of Müller et al. ${ }^{33}$ this spectrum strongly evokes the $\mathrm{FAD}^{\bullet-} / \mathrm{W}^{\bullet}$ pair, resulting from deprotonation 
of the terminal $\mathrm{WH}^{\bullet+}$ radical. This interpretation is supported by a large kinetic isotope effect $\left(\mathrm{KIE}=k_{\mathrm{H}} / k_{\mathrm{D}}\right)$ of 2.5 for time constant $\tau_{3}$ (Table 1$)$. The $\tau_{3}$ constants $\left(230 \mathrm{ps}\right.$ in $\mathrm{H}_{2} \mathrm{O}$ and $570 \mathrm{ps}$ in $\mathrm{D}_{2} \mathrm{O}$ ) are somewhat shorter than those reported by Müller et al. (350 and $800 \mathrm{ps}$ ) but should be considered as essentially compatible within the experimental errors. Notably, while the reference spectrum of deprotonated tryptophanyl radical $\mathrm{W}^{\bullet}$ drops to zero above $600 \mathrm{~nm},{ }^{48}$ we observe a weak absorption tail extending EADS4 down to $700 \mathrm{~nm}$. This tail corresponds well to the red extension of the $\mathrm{FAD}^{\bullet-}$ spectrum reported by Berndt et $a l .{ }^{46}$ and is tentatively assigned to this species.

\subsection{In-depth analysis of the EADS (WT in $\mathrm{H}_{2} \mathrm{O}$ )}

\subsubsection{EADS3}

It appears from the above inspection of the spectroscopic products that EADS2 and EADS3 are essentially similar, the main difference being an additional dip around $533 \mathrm{~nm}$ in EADS2. This is why the simpler looking EADS3 will be analyzed first. As explained in $\S 2.5$ we tried to fit it with a linear combination of reference spectra of $\mathrm{FAD}^{\bullet-}$ and $\mathrm{WH}^{\bullet+}$, suitably scaled and shifted, and complemented by the negative $\mathrm{FAD}_{\mathrm{ox}}$ bleaching contribution. The optimized parameters, the modified reference spectra and the fitting equation may be found in ESI-§4.2. The modification imposed on the reference spectra (Figure S8A) are relatively modest and compatible with the comments made above (expected red shift of the $\mathrm{WH}^{\bullet+}$ spectrum). The final result, shown in Figure 4A, strongly supports the hypothesis that EADS3 indeed characterizes the product of a photoinduced electron transfer from a tryptophan residue to FAD.

The fit of EADS3 could in fact be considerably improved ( $\mathrm{R}^{2}$ going from 0.9929 to 0.9972$)$ by adding a $\mathrm{YO}^{\bullet}$ component, to reflect the oxidation of a tyrosine residue. It is however difficult to 
ascertain that this corresponds to any real contribution by the $\mathrm{YO}^{\bullet}$ radical because both $\mathrm{FAD}^{\bullet}$ and $\mathrm{YO}^{\bullet}$ have a local maximum at around $400 \mathrm{~nm}$ (see ESI- $\$ 4.1$ ). As the $\mathrm{YO}^{\bullet}$ component may be used during the fit as a mere modifier of the $\mathrm{FAD}^{\bullet-}$ spectrum, this improved fit was not retained.

\subsubsection{Difference between EADS2 and EADS3}

Next, we explore the idea that the difference between EADS2 and EADS3 may be caused by a contribution from $\mathrm{FAD}_{\mathrm{ox}}{ }^{*}$. We could indeed fit EADS3 very well $\left(\mathrm{R}^{2}=0.9982\right)$ by a weighted sum of EADS2 and a slightly modified EADS1 (Figure 4B; ESI-§4.3), namely $\mathrm{EADS} 3 \approx 0.61 \mathrm{EADS} 2-0.08 \mathrm{EADS} 1{ }^{\prime} . \mathrm{EADS} 1$ ' is here taken as a representative spectrum of $\mathrm{FAD}_{\mathrm{ox}}{ }^{*}$. According to this fit, a rather large loss of the $\mathrm{FAD}^{\bullet-} / \mathrm{WH}^{\bullet+}$ population $(39 \%)$ happens during the 29-ps phase (EADS2 $\rightarrow$ EADS3) without any modification of the corresponding isotropic spectrum apart from the decay of a residual population of $\mathrm{FAD}_{\mathrm{ox}} *$. This conversely tells us that EADS2 still comprises a residual fraction of unreacted $\mathrm{FAD}_{\mathrm{ox}}{ }^{*}$, the origin of which is yet unclear. Similar results were obtained for all systems under study. Free FAD in solution seems unlikely because the samples were gel-filtrated immediately before each experiment to remove such species. In addition, EADS1', as EADS1, is highly characteristic of a bound flavin, its SE band being in particular considerably more blue shifted than that of free FAD. ${ }^{44}$ Currently, one may hypothesize the presence of a minority population of $M m \mathrm{CPDII}$ that exhibits a larger distance between FAD and the proximal tryptophan. Note that multiexponential $\mathrm{FAD}_{\mathrm{ox}} *$ decays have repeatedly been observed in BLUF proteins, caused by a FAD-tyrosine electron transfer, and explained in a similar way. ${ }^{50-52}$

\subsubsection{Difference between EADS3 and EADS4}


We next fitted EADS4 with a weighted sum of EADS3, $\mathrm{W}^{\bullet}$ and $\mathrm{WH}^{\bullet+}$ contributions, as detailed in ESI-§4.4 (Equation S3). As above, this approach focuses on the difference between EADS3 and EADS4 once initial state recovery is taken into account. The result shown in Figure 4C (see optimized parameters and modified reference spectra in ESI-§4.4) provides a convincing support that $\mathrm{WH}^{\bullet+}$ indeed undergoes deprotonation during the last kinetic step (230 ps), as qualitatively deduced in $\S 4.1$. The fit also indicates that the deprotonation process occurs in competition with charge recombination during this phase, the latter process concerning about $30 \%$ of the transient population. As previously commented for other photolyases, ${ }^{22,27}$ it is likely that charge recombination during all ps phases mostly takes place from the proximal $\mathrm{FAD}^{\bullet}$ $/ \mathrm{W}_{381} \mathrm{H}^{\bullet+}$ pair, because the radicals are here separated by a short distance (5.3 $\AA$; Figure 1). For the same reason, recombination involving more distant partners is expected to be quite ineffective in this timescale. Combining the charge recombination losses of kinetic steps 2 and 3 (and ignoring possible excited-state deactivation through electron transfer from the adenine moiety of FAD during step 1 , as previously reported ${ }^{22}$ ), the total quantum yield of charge separation at the end of step 3 can be estimated to $43 \%$, which is rather close to the value of $\sim 55 \%$ derived differently by Müller $e t a l^{33}$

Interestingly, a specific region of EADS4, around $400 \mathrm{~nm}$, is not well reproduced by this spectral fit (Figure 4C). A better fit ( $\mathrm{R}^{2}$ going from 0.9939 to 0.9958$)$ could be again obtained with an additional $\mathrm{YO}^{\bullet}$ component (see Equation S4). This fit (for optimized parameters and modified reference spectra refer to ESI-§4.4) exhibits a significantly better reproduction of the region around $400 \mathrm{~nm}$ (Figure 4D). Here, the $\mathrm{YO}^{\bullet}$ spectrum shows no overlap with any other reference spectrum of the sum and may be hence further considered. A contribution of the $\mathrm{YO}^{\bullet}$ species to EADS4 implies the occurrence of electron transfer from a tyrosine residue to a $\mathrm{WH}^{\bullet+}$ 
radical in competition with $\mathrm{WH}^{\bullet+}$ deprotonation and charge recombination. The observation of $\mathrm{YO}^{\bullet}$ also implies that the protonated $\mathrm{YOH}^{\bullet+}$ radical $^{53}$ expected to be produced by this transfer would very rapidly (or concomitantly) deprotonate, in agreement with the extreme acidity of this

species $(\mathrm{pKa} \sim-2) .{ }^{54}$ From the above, we cannot however infer whether any $\mathrm{YO}^{\bullet}$ species already contributes to EADS2 or EADS3. Similar results are obtained for $M m C P D I I$ in $\mathrm{D}_{2} \mathrm{O}$ buffer. Given the particularly short distance between $\mathrm{Y}_{345}$ and $\mathrm{W}_{388}(3.7 \AA$; see Figure 1$)$ and the established involvement of $\mathrm{Y}_{345}$ in the ms dynamics of MmCPDII, ${ }^{33}$ it may be hypothesized that $\mathrm{Y}_{345}$ acts as electron donor and $\mathrm{W}_{388} \mathrm{H}^{\bullet+}$ as electron acceptor on the fast time scale. The following section compares therefore the results of WT and Y345F in more details.

\subsection{Comparison of WT and Y345F}

Although the raw transient absorption spectra of WT and Y345F appear at first sight identical, subtle differences appear when one superposes them. Figure 5 compares the EADS of both molecules, normalized at the maximum of their bleaching band (NEADS) of around $445 \mathrm{~nm}$. One observes a near perfect overlap of NEADS1 (Figure 5A), which is expected as it corresponds to the same initial excited state of $\mathrm{FAD}_{\mathrm{ox}}$ and shows that WT and Y345F spectra may be quantitatively compared. However, a subtle difference can be noted when comparing the final NEADS4 (Figure 5B). Here, a slightly more intense relative value appears at around 404 $\mathrm{nm}$ for WT, while a somewhat diminished intensity is found between 510 and $610 \mathrm{~nm}$. Given the quality of the superposition of NEADS1, it is likely that these mismatches are significant. We propose that $\mathrm{Y}_{345}$ is indeed involved in the last kinetic step and reduces the nearby $\mathrm{W}_{388} \mathrm{H}^{\bullet+}$ radical to a small extent, in competition with the overwhelming deprotonation of the latter. Compared to $\mathrm{Y} 345 \mathrm{~F}$, this additional reaction pathway is indeed expected in WT to increase the relative intensity of EADS4 around $400 \mathrm{~nm}$ because of the presence of $\mathrm{YO}^{\circ}$ and to reduce the 
intensity in the red part of the spectrum because of the slightly reduced yield of production of $\mathrm{W}^{\bullet}$. In agreement with this, the spectral analysis of the EADS4 of Y345F did not suggest the additional presence of any $\mathrm{YO}^{\bullet}$ contribution (see ESI-§4.5).

The hypothesis of the involvement of $\mathrm{Y}_{345}$ in WT is further supported by the smaller value (by a factor of 1.5) of the last time constant in comparison to $\mathrm{Y} 345 \mathrm{~F}\left(\tau_{3}\right.$, Table 1). This is in qualitative agreement with the presence of an additional reaction channel, namely electron transfer from $\mathrm{Y}_{345}$ to $\mathrm{W}_{388} \mathrm{H}^{\bullet+}$, in WT. On the basis of the fit parameters (ESI-§4.4, Table S4), the quantum yield of $Y_{345}$ oxidation during the 230-ps phase may be roughly estimated to $\sim 25 \%$, which would set the corresponding rate to $\sim 1 \mathrm{~ns}^{-1}$ (see ESI-§4.4).

Strikingly, the oxidation of $\mathrm{Y}_{345}$ by $\mathrm{W}_{388} \mathrm{H}^{\bullet+}$ could take place in the ns regime while the oxidation of $\mathrm{Y}_{345}$ by $\mathrm{W}_{388^{\bullet}}$ is reported to occur in $\sim 1 \mathrm{~ms}$ by Müller et al..$^{33}$ In the latter study it was suggested that, in the ms timescale, $\mathrm{Y}_{345}$ is mostly oxidized by $\mathrm{W}_{388} \mathrm{H}^{\bullet+}$, which is present in only minor amounts under acid-base equilibrium with $\mathrm{W}_{388^{\bullet}}$ (governed by the $\mathrm{pKa}$ of $\mathrm{W}_{388} \mathrm{H}^{\bullet+}$ ). Byrdin et al..$^{55}$ developed a theoretical treatment of this type of equilibrium in the context of charge recombination. Using this model and assuming that $\mathrm{pH} \gg>\mathrm{pKa}$, the rate for $\mathrm{Y}_{345}$ oxidation under acid-base equilibrium reduces to:

$$
k_{e q}=k_{1} 10^{-(p H-p K a)}+k_{2}
$$

where $\mathrm{k}_{1}$ stands for the oxidation rate caused by $\mathrm{W}_{388} \mathrm{H}^{\bullet+}$ and $\mathrm{k}_{2}$ for the rate caused by $\mathrm{W}_{388}$. Setting $\mathrm{k}_{\mathrm{eq}}$ to $\sim 1 \mathrm{~ms}^{-1}$ and $\mathrm{k}_{1}$ to $\sim 1 \mathrm{~ns}^{-1}$, one deduces that the $\mathrm{pH}$-pKa difference is larger than 6 (which justifies the assumption yielding Equation 3). Given that Müller et al.'s study was conducted at $\mathrm{pH} 8.0$, this implies an $\mathrm{pKa}$ of $<2$ for the $\mathrm{W}_{388} \mathrm{H}^{\bullet+}$ radical cation. For reference, the pKa of $\mathrm{W}_{306} \mathrm{H}^{\bullet+}$ of the class I photolyase of $E$. coli $(E c \mathrm{CPDI})$ was estimated to $4.0 .^{55}$ The 
much lower $\mathrm{pKa}$ of $\mathrm{W}_{388} \mathrm{H}^{\bullet+}$ in $M m$ CPDII is fully consistent with the observation that $\mathrm{W}_{388} \mathrm{H}^{\bullet+}$ deprotonates three orders of magnitude faster in $M m$ CPDII than $\mathrm{W}_{306} \mathrm{H}^{\bullet+}$ in $E c$ CPDI.

\subsection{Analysis of the transient anisotropy data}

In this section, we use the anisotropy data to try to determine the residues responsible for the $\mathrm{WH}^{\bullet+}$ signature that is detected in our transient absorption spectra. Following an approach previously outlined in Ref. ${ }^{27}$, we chose to probe the maximum of $\mathrm{WH}^{\bullet+}$ around $600 \mathrm{~nm}$, expecting under these conditions that essentially a single transition of $\mathrm{WH}^{\bullet+}$ is probed. Table 2 gathers values of the EAAS (see $\$ 2.4)$ at $600 \mathrm{~nm}$ (averaged over $6 \mathrm{~nm}$ ), after the initial decay of the excited state.

\subsubsection{Correction of EAAS2}

From the spectral analysis of $\S 4.3$, we found that the isotropic EADS2 is well represented by a linear combination of EADS1 and EADS3 (the small modification of EADS1 used in $\S 4.3$ may be dropped without major drawback). The relation $\mathrm{EADS} 2 \approx \mathrm{a} \times \mathrm{EADS} 1+\mathrm{b} \times \mathrm{EADS} 3$ is however not well preserved for the respective polarized EADS as it yielded only unsatisfactory fits. To explain this discrepancy one may argue that the anisotropy of the $\mathrm{FAD}_{\mathrm{ox}}{ }^{*}$ contribution to EADS2 is different from that of EADS1, as for instance if part of the initially excited flavins had undergone a rotation or had transferred their excitation to different flavins (e.g. belonging to another protein of an aggregate). However, a more likely explanation that is consistent with the structural context of the chromophoric groups within $M m$ CPDII is that the anisotropy of the $\mathrm{WH}^{\bullet+}$ contribution to EADS2 differs from that of EADS3, because the oxidation hole migrates along the tryptophan triad during the EADS2 $\rightarrow$ EADS3 step. 
When following the latter notion, one can set the anisotropy of the $\mathrm{FAD}_{\mathrm{ox}}$ * contribution to EADS2 to be equal to that of EADS1. It is then legitimate to subtract a $\times$ EADS1 $1_{\|}$from EADS2 $\|$ and $\mathrm{a} \times \mathrm{EADS} 1_{\perp}$ from $\mathrm{EADS} 2_{\perp}$ in order to get corrected versions of the polarized EADS2, which are free from any $\mathrm{FAD}_{\mathrm{ox}}{ }^{*}$ contribution. From those one deduces a corrected EAAS2 (EAAS2') as shown in Figure 6 for WT in $\mathrm{H}_{2} \mathrm{O}$ buffer. Interestingly, EAAS2' lacks any suspicious dip around $535 \mathrm{~nm}$. Instead, a rather flat profile is obtained that resembles the shape of EAAS3 in this region but is conspicuously displaced towards positive values; no major changes are observed below $500 \mathrm{~nm}$. This result, similarly obtained for all systems under study supports the idea that the main difference between EAAS2' and EAAS3 is the anisotropy of the $\mathrm{WH}^{\bullet+}$ contribution in the red. The above reasoning supports the idea that the $\mathrm{FAD}_{\mathrm{ox}}{ }^{*}$ population contributing to EADS2 comes from a sub-pool of the initially excited $\mathrm{FAD}_{\mathrm{ox}}$ molecules that is possibly characterized by a larger distance between FAD and the proximal tryptophan. The corrected anisotropies at $600 \mathrm{~nm}\left(\mathrm{r}_{2}{ }^{\prime}\right)$ are reported in Table 2.

\subsubsection{Theoretical predictions}

At this point, it is interesting to compare the experimental anisotropies to predictions based on the crystal structure of $M m \mathrm{CPDII}$ (PDB entry $2 \mathrm{XRY})^{7}$ and on known directions of the transition dipole moments of $\mathrm{FAD}_{\mathrm{ox}}$ and $\mathrm{WH}^{\bullet+}$, as previously described. ${ }^{27}$ The expected anisotropies at 600 $\mathrm{nm}$ for the different $\mathrm{WH}^{\bullet+}$ radicals of the photoreduction chain are shown in Table 3 (line raw). Errors indicated in small characters correspond to variations of $\pm 5^{\circ}$ of the directions of both $\mathrm{FAD}_{\mathrm{ox}}$ and $\mathrm{WH}^{\bullet+}$ transition moments within the molecular plane.

These predictions may however not be fully comparable to the experimental anisotropies because, as mentioned in $\S 4.1$, a small contribution of $\mathrm{FAD}^{\bullet-}$ could be present at $600 \mathrm{~nm}$. The intrinsic anisotropy of $\mathrm{FAD}^{\bullet-}$ at $600 \mathrm{~nm}$ can be roughly estimated by taking EAAS4 of Y345F 
(less noisy than that of WT; Figure 3D) and assuming that the population of $\mathrm{WH}^{\bullet+}$, mostly deprotonated at the plateau, is negligible. Since neither $\mathrm{W}^{\bullet}$ nor $\mathrm{YO}^{\bullet}$ absorb at $600 \mathrm{~nm}$, the only remaining contribution would be that of $\mathrm{FAD}^{\bullet-}$. On this basis, a value of the order of -0.2 is deduced for of $\mathrm{FAD}^{\bullet-}$. If both $\mathrm{WH}^{\bullet+}$ and $\mathrm{FAD}^{\bullet-}$ contribute to the experimental anisotropy, the latter should be decomposed as:

$$
r_{\exp }=\mathrm{xr}_{\mathrm{W}}+(1-\mathrm{x}) \mathrm{r}_{\mathrm{F}}
$$

where $\mathrm{r}_{\mathrm{W}}$ and $\mathrm{r}_{\mathrm{F}}$ are the pure anisotropies respectively attached to $\mathrm{WH}^{\bullet+}$ and $\mathrm{FAD}^{\bullet-}$ and $\mathrm{x}$ is the relative contribution of $\mathrm{WH}^{\bullet+}$ to the total isotropic signal at $600 \mathrm{~nm}$. Taking as an approximate lower limit $\mathrm{x}=0.8$ (a value of 0.83 may be obtained from the modified $\mathrm{WH}^{\bullet+}$ and $\mathrm{FAD}^{\bullet-}$ spectra of Figure S8; see ESI- $\$ 4.2$ ), one can delineate corrected values (Table 3, line corrected), which serve as an indicator for the effect of the $\mathrm{FAD}^{\bullet-}$ contribution to the predictions.

This analysis shows first that the corrected experimental value for $r_{2}^{\prime}(0.073)$ is rather close (within error) to the predicted anisotropy of $\mathrm{W}_{381} \mathrm{H}^{\bullet+}(0.05)$. This is consistent with the fact that the primary reductant of the excited flavin is the proximal $\mathrm{W}_{381}$. The possible role of $\mathrm{W}_{421}$ as alternative electron donor for $\mathrm{FAD}_{\mathrm{ox}}$ * can be discarded despite its similar proximity (see Figure 1), because the expected anisotropy is slightly negative for $\mathrm{W}_{421} \mathrm{H}^{\bullet+}$ (Table 3). No trace of the highly positive anisotropy that would be characteristic for $\mathrm{W}_{360} \mathrm{H}^{\bullet+}$ formation is visible in the experimental anisotropy data. Instead, $\mathrm{r}_{3}$ is closer to the negative anisotropy expected for $\mathrm{W}_{388} \mathrm{H}^{\bullet+}$ although the match is far from perfect. The difficulty to assign $r_{3}$ could of course be attributed to the insufficient quality of the predictions. It is however also possible that EAAS3 actually receives contributions from a mixture of $\mathrm{WH}^{\bullet+}$ radicals, meaning that at this step of the reaction the oxidation hole is in fact delocalized over medial and distal tryptophan residues $\left(\mathrm{W}_{360}\right.$ and $\mathrm{W}_{388}$ ). Delocalization is here understood as the result of a population equilibrium, involving 
forward and backward-directed electron transfer. $\mathrm{W}_{381}$ may participate in this delocalization, even to a small extent, because charge recombination still occurs during the 230-ps step and the $\mathrm{FAD}^{\bullet-} / \mathrm{W}_{381} \mathrm{H}^{\bullet+}$ pair is the most effective channel for it due to the short distance between the charges. A similar conclusion was previously drawn for the photoreduction of oxidized $X l 64$, for which the final localization of the oxidation hole on the last tryptophan was supposed to require several hundreds of ps. $^{27}$

In the present case, sub-ns deprotonation of $\mathrm{W}_{388} \mathrm{H}^{\bullet+}$ occurs during the last kinetic step, before this radical localizes on the distal tryptophan. It may however be observed that the anisotropy of the plateau (EAAS4) taken at $535 \mathrm{~nm}$, where one can expect that the contribution of $\mathrm{W}^{\bullet}$ is dominant (see Figure S7), has a value of $c a$. -0.15 (Figure 3C for WT). On the other hand, one may calculate the expected anisotropy for the lowest transition of $\mathrm{W}^{\bullet}$. The obtained values are in fact quite close to the ones reported for $\mathrm{WH}^{\bullet+}$ in Table 3 because the orientation of the transition dipole moment of $\mathrm{W}^{\bullet}\left(104^{\circ}\right.$ from short axis $\left.{ }^{34}\right)$ is similar to that of $\mathrm{WH}^{\bullet+}\left(108^{\circ 27}\right)$. The raw calculation for $\mathrm{W}_{388}{ }^{\bullet}$ is -0.17 , which is proximate to the experimental value. A correction for the contribution of $\mathrm{FAD}^{\bullet-}$ would be necessary but is rather uncertain to apply because of the possible involvement of different transitions at $532 \mathrm{~nm}$. We then assume it would not be large, as for $\mathrm{W}_{388} \mathrm{H}^{\bullet+}$ in Table 3, and take the above approximate match as a plausible suggestion that the final localization of the oxidation hole of the distal tryptophan is complete after the 230-ps deprotonation step.

\section{CONCLUSIONS}


The transient absorption results presented here permit to draw the following image of the primary events occurring upon excitation of the $\mathrm{FAD}_{\text {ox }}$ cofactor of $M m \mathrm{CPDII}$ (Figure 7). The first event is an electron transfer from a tryptophan residue to the excited flavin, occurring with a time constant of $9.2 \mathrm{ps}$ and yielding a $\mathrm{FAD}^{\bullet-} / \mathrm{WH}^{\bullet+}$ pair of radicals in their ground state. This time constant is notably $\sim 20$ times slower than for the homologous reaction of other known oxidized photolyases or cryptochromes. ${ }^{21,22,24,25,27,28}$ The difference is mostly assigned to the large distance in $\mathrm{MmCPDII}$ between the flavin and the proximal tryptophan, $\mathrm{W}_{381}$, as class II photolyases depend on an entirely different tryptophan triad than most other photolyases and cryptochromes. ${ }^{7,33}$ Our anisotropy measurements confirm the assignment of $\mathrm{W}_{381} \mathrm{H}^{\bullet+}$ as the primary oxidized species formed within the 9.2 ps phase. Furthermore, it appears that not all of the excited flavins are reduced during the initial phase and that a small fraction of them only decays during the second kinetic phase, in 29 ps. The nature of this slowly reacting $\mathrm{FAD}_{\mathrm{ox}}{ }^{*}$ population is currently uncertain. The second kinetic phase is however mainly characterized by a partial decay of the transient absorption signal, assigned to charge recombination of the $\mathrm{FAD}^{\circ}$ $/ \mathrm{WH}^{\bullet+}$ pair, and by a change of the anisotropy signature of $\mathrm{WH}^{\bullet+}$ at $600 \mathrm{~nm}$, due to the migration of the oxidation hole along the tryptophan triad. An anisotropy found to be somehow intermediate between the values expected for the medial $\mathrm{W}_{360} \mathrm{H}^{\bullet+}$ and distal $\mathrm{W}_{388} \mathrm{H}^{\bullet+}$ further suggests that the oxidation hole quickly delocalizes over various tryptophans by population equilibrium, thereby implying the existence of efficient backward-directed electron transfer reactions. Contrary to other photolyases where $\mathrm{WH}^{\bullet+}$ localizes on the distal tryptophan of the triad before slowly deprotonating, ${ }^{27,34}$ deprotonation is here seen to occur in $230 \mathrm{ps}$, while $\mathrm{WH}^{\bullet+}$ is still delocalized. Anisotropy data suggest that localization of the oxidation hole on the distal tryptophan might be reached after this step. Tryptophanyl deprotonation occurs in competition 
with charge recombination, but also with a minor channel for electron transfer from a tyrosine residue to $\mathrm{WH}^{\bullet+}$ radical. We found that $\mathrm{Y}_{345}$, previously reported to be oxidized by the neutral $\mathrm{W}_{388^{\circ}}$ radical in the ms timescale is oxidized much more rapidly, in the ns timescale, by the still protonated $\mathrm{W}_{388} \mathrm{H}^{\bullet+}$ radical cation.

\section{CONFLICT OF INTERESTS}

There are no conflicts to declare.

\section{ACKNOWLEDGEMENTS}

This work was supported by the Air Force Office of Scientific Research (AFOSR; grant FA9550-14-1-0409).

\section{REFERENCES}

1. A. Sancar, Chem. Rev., 2003, 103, 2203-2237.

2. S. Weber, Biochim. Biophys. Acta Bioenerg., 2005, 1707, 1-23.

3. M. Müller and T. Carell, Curr. Opin. Struct. Biol., 2009, 19, 277-285.

4. K. Brettel and M. Byrdin, Curr. Opin. Struct. Biol., 2010, 20, 693-701.

5. J. Yamamoto, P. Plaza and K. Brettel, Photochem. Photobiol., 2017, 93, 51-66.

6. J. Cadet, E. Sage and T. Douki, Mutation Res., 2005, 571, 3-17.

7. S. Kiontke, Y. Geisselbrecht, R. Pokorny, T. Carell, A. Batschauer and L.-O. Essen, EMBO J., 2011, 30, 4437-4449.

8. Y. Geisselbrecht, S. Fruehwirth, C. Schroeder, A. J. Pierik, G. Klug and L.-O. Essen, EMBO Rep., 2012, 13, 223-229.

9. M. Juhas, A. von Zadow, M. Spexard, M. Schmidt, T. Kottke and C. Buechel, FEBS J., 2014, 281, 2299-2311.

10. P. Oliveri, A. E. Fortunato, L. Petrone, T. Ishikawa-Fujiwara, Y. Kobayashi, T. Todo, O. Antonova, E. Arboleda, J. Zantke, K. Tessmar-Raible and A. Falciatore, Mar. Genom., 2014, 14, 23-37. 
11. A. Okafuji, T. Biskup, K. Hitomi, E. D. Getzoff, G. Kaiser, A. Batschauer, A. Bacher, J. Hidema, M. Teranishi, K. Yamamoto, E. Schleicher and S. Weber, DNA Repair, 2010, 9, 495-505.

12. K. Hitomi, A. S. Arvai, J. Yamamoto, C. Hitomi, M. Teranishi, T. Hirouchi, K. Yamamoto, S. Iwai, J. A. Tainer, J. Hidema and E. D. Getzoff, J. Biol. Chem., 2012, 287, 12060-12069.

13. S. Kiontke, P. Gnau, R. Haselsberger, A. Batschauer and L.-O. Essen, J. Biol. Chem., 2014, 289, 19659-19669.

14. E. Ignatz, Y. Geisselbrecht, S. Kiontke and L. O. Essen, Photochem. Photobiol., 2018, 94, 81-87.

15. M. Zhang, L. J. Wang, S. Shu, A. Sancar and D. P. Zhong, Science, 2016, 354, 209-213.

16. C. T. Lin and D. Shalitin, Annu. Rev. Plant Biol., 2003, 54, 469-496.

17. I. Chaves, R. Pokorny, M. Byrdin, N. Hoang, T. Ritz, K. Brettel, L.-O. Essen, G. T. J. van der Horst, A. Batschauer and M. Ahmad, Annu. Rev. Plant Biol., 2011, 62, 335-364.

18. C. Aubert, M. H. Vos, P. Mathis, A. P. M. Eker and K. Brettel, Nature, 2000, 405, 586590.

19. A. Lukacs, A. P. M. Eker, M. Byrdin, K. Brettel and M. H. Vos, J. Am. Chem. Soc., 2008, 130, 14394-14395.

20. M. Byrdin, A. Lukacs, V. Thiagarajan, A. P. M. Eker, K. Brettel and M. H. Vos, J. Phys. Chem. A, 2010, 114, 3207-3214.

21. H. Y. Wang, C. Saxena, D. H. Quan, A. Sancar and D. P. Zhong, J. Phys. Chem. B, 2005, 109, 1329-1333.

22. Z. Y. Liu, C. Tan, X. M. Guo, J. Li, L. J. Wang, A. Sancar and D. P. Zhong, Proc. Natl. Acad. Sci. USA, 2013, 110, 12966-12971.

23. Z. Y. Liu, C. Tan, X. M. Guo, J. Li, L. J. Wang and D. P. Zhong, J. Phys. Chem. Lett., 2014, 5, 820-825.

24. Y. T. Kao, C. Tan, S. H. Song, N. Ozturk, J. Li, L. J. Wang, A. Sancar and D. P. Zhong, J. Am. Chem. Soc., 2008, 130, 7695-7701.

25. J. Brazard, A. Usman, F. Lacombat, C. Ley, M. M. Martin, P. Plaza, L. Mony, M. Heijde, G. Zabulon and C. Bowler, J. Am. Chem. Soc., 2010, 132, 4935-4945.

26. P. Müller, J. Yamamoto, R. Martin, S. Iwai and K. Brettel, Chem. Commun., 2015, 51, 15502-15505.

27. R. Martin, F. Lacombat, A. Espagne, N. Dozova, P. Plaza, J. Yamamoto, P. Müller, K. Brettel and A. de la Lande, Phys. Chem. Chem. Phys., 2017, 19, 24493-24504.

28. D. Immeln, A. Weigel, T. Kottke and J. L. Perez Lustres, J. Am. Chem. Soc., 2012, 134, 12536-12546.

29. P. Müller, J.-P. Bouly, K. Hitomi, V. Balland, E. D. Getzoff, T. Ritz and K. Brettel, Sci. Rep., 2014, 4, 5175.

30. C. Aubert, P. Mathis, A. P. M. Eker and K. Brettel, Proc. Natl. Acad. Sci. USA, 1999, 96, 5423-5427.

31. B. Giovani, M. Byrdin, M. Ahmad and K. Brettel, Nat. Struc. Biol., 2003, 10, 489-490.

32. D. Nohr, S. Franz, R. Rodriguez, B. Paulus, L. O. Essen, S. Weber and E. Schleicher, Biophys. J., 2016, 111, 301-311.

33. P. Müller, E. Ignatz, S. Kiontke, K. Brettel and L. O. Essen, Chem. Sci., 2018, 9, 12001212. 
34. M. Byrdin, S. Villette, A. Espagne, A. P. M. Eker and K. Brettel, J. Phys. Chem. B, 2008, 112, 6866-6871.

35. P. Macheroux, in Flavoprotein Protocols, eds. S. K. Chapman and G. A. Reid, 1999, pp. 17.

36. A. Yoshimura, M. Z. Hoffman and H. Sun, J. Photochem. Photobiol. A-Chem., 1993, 70, 29-33.

37. M. Byrdin, V. Thiagarajan, S. Villette, A. Espagne and K. Brettel, Rev. Sci. Instr., 2009, 80, 043102.

38. K. Ekvall, P. van der Meulen, C. Dhollande, L. E. Berg, S. Pommeret, R. Naskrecki and J. C. Mialocq, J. Appl. Phys., 2000, 87, 2340-2352.

39. I. H. M. van Stokkum, S. L. Delmar and R. van Grondelle, Biochim. Biophys. Acta Bioenerg., 2004, 1657, 82-104.

40. E. R. Henry and J. Hofrichter, Methods Enzymol., 1992, 210, 129-193.

41. A. Weigel, A. Dobryakov, B. Klaumunzer, M. Sajadi, P. Saalfrank and N. P. Ernsting, J. Phys. Chem. B, 2011, 115, 3656-3680.

42. J. Zhu, T. Mathes, Y. Hontani, M. T. A. Alexandre, K. C. Toh, P. Hegemann and J. T. M. Kennis, J. Phys. Chem. Lett., 2016, 7, 4380-4384.

43. J. P. Hoben, C. E. Lubner, M. W. Ratzloff, G. J. Schut, D. M. N. Nguyen, K. W. Hempel, M. W. W. Adams, P. W. King and A.-F. Miller, J. Biol. Chem., 2017, 292, 14039-14049.

44. J. Brazard, A. Usman, F. Lacombat, C. Ley, M. M. Martin and P. Plaza, J. Phys. Chem. A, 2011, 115, 3251-3262.

45. V. Massey and G. Palmer, Biochemistry, 1966, 5, 3181-3189.

46. A. Berndt, T. Kottke, H. Breitkreuz, R. Dvorsky, S. Hennig, M. Alexander and E. Wolf, J. Biol. Chem., 2007, 282, 13011-13021.

47. B. Liu, H. Liu, D. Zhong and C. Lin, Curr. Opin. Plant Biol., 2010, 13, 578-586.

48. S. Solar, N. Getoff, P. S. Surdhar, D. A. Armstrong and A. Singh, J. Phys. Chem., 1991, 95, 3639-3643.

49. C. C. Moser and P. L. Dutton, Biochim. Biophys. Acta, 1992, 1101, 171-176.

50. M. Gauden, I. H. M. van Stokkum, J. M. Key, D. C. Luehrs, R. Van Grondelle, P. Hegemann and J. T. M. Kennis, Proc. Natl. Acad. Sci. USA, 2006, 103, 10895-10900.

51. C. Bonetti, M. Stierl, T. Mathes, I. H. M. van Stokkum, K. M. Mullen, T. A. Cohen-Stuart, R. van Grondelle, P. Hegemann and J. T. M. Kennis, Biochemistry, 2009, 48, 1145811469.

52. T. Mathes, I. H. M. van Stokkum, M. Stierl and J. T. M. Kennis, J. Biol. Chem., 2012, 287, 31725-31738.

53. L. Nag, P. Sournia, H. Myllykallio, U. Liebl and M. H. Vos, J. Am. Chem. Soc., 2017, 139, 11500-11505.

54. W. T. Dixon and D. Murphy, J. Chem. Soc. Faraday Trans., 1976, 72, 1221-1230.

55. M. Byrdin, V. Sartor, A. P. M. Eker, M. H. Vos, C. Aubert, K. Brettel and P. Mathis, Biochim. Biophys. Acta Bioenerg., 2004, 1655, 64-70. 


\section{FIGURES}

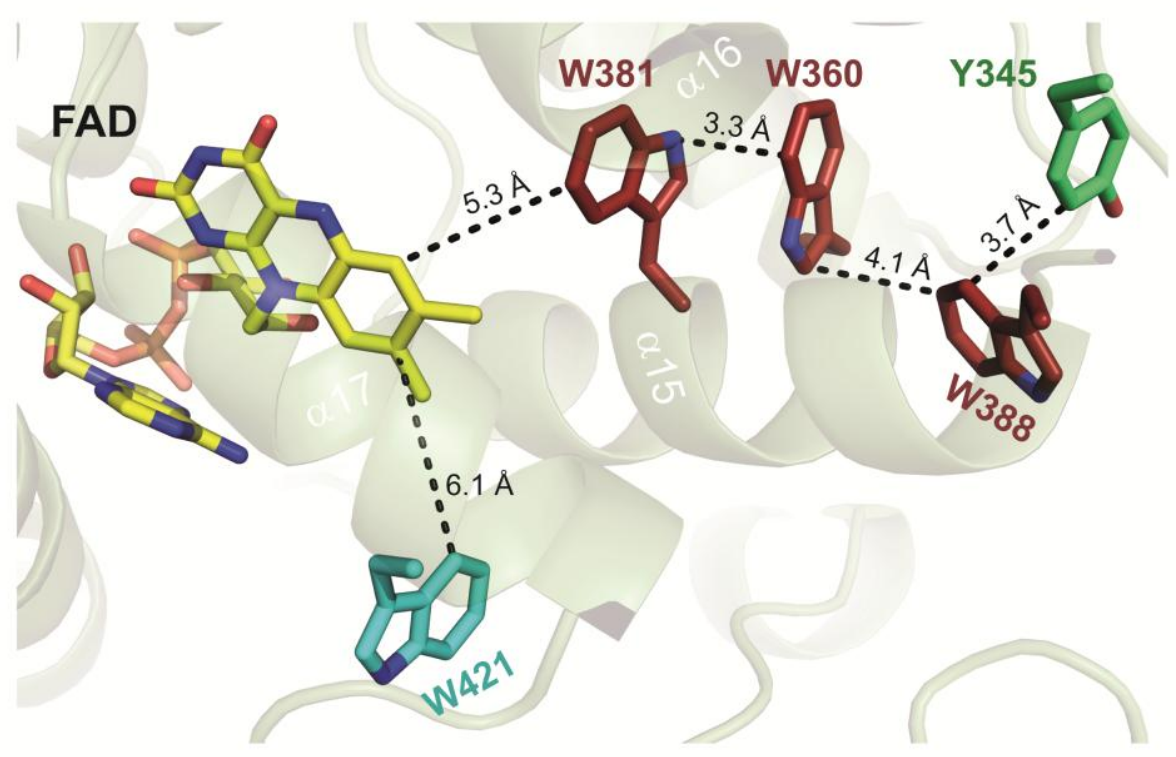

Figure 1. Structure of $M m$ CPDII in the flavin photoreduction site (from PDB entry $2 \mathrm{XRY}$ ). FAD is represented in yellow, the tryptophan triad $\left(\mathrm{W}_{381}, \mathrm{~W}_{360}, \mathrm{~W}_{388}\right)$ in red and $\mathrm{Y}_{345}$ in green. An additional tryptophan $\left(\mathrm{W}_{421}\right)$, located close to $\mathrm{FAD}$, is indicated in cyan. Closest edge-to-edge distances between aromatic moieties are indicated. 

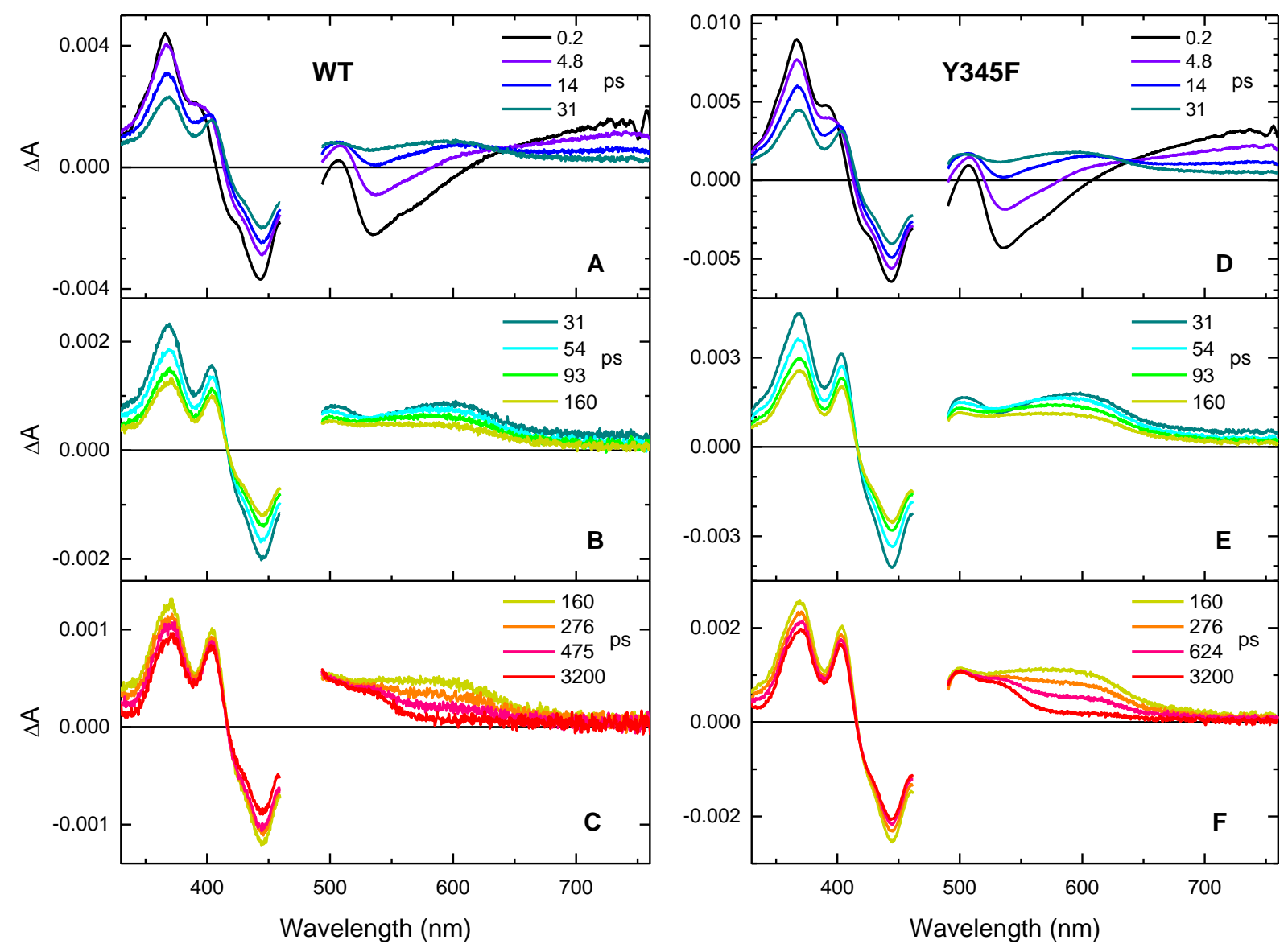

Figure 2. Isotropic transient absorption spectra of $M m C P D I I\left(\mathrm{H}_{2} \mathrm{O}\right.$ buffer $)$, at selected pumpprobe delays, after fs excitation at $475 \mathrm{~nm}$. WT is shown in panels A-C, the Y345F mutant in panels D-F. 

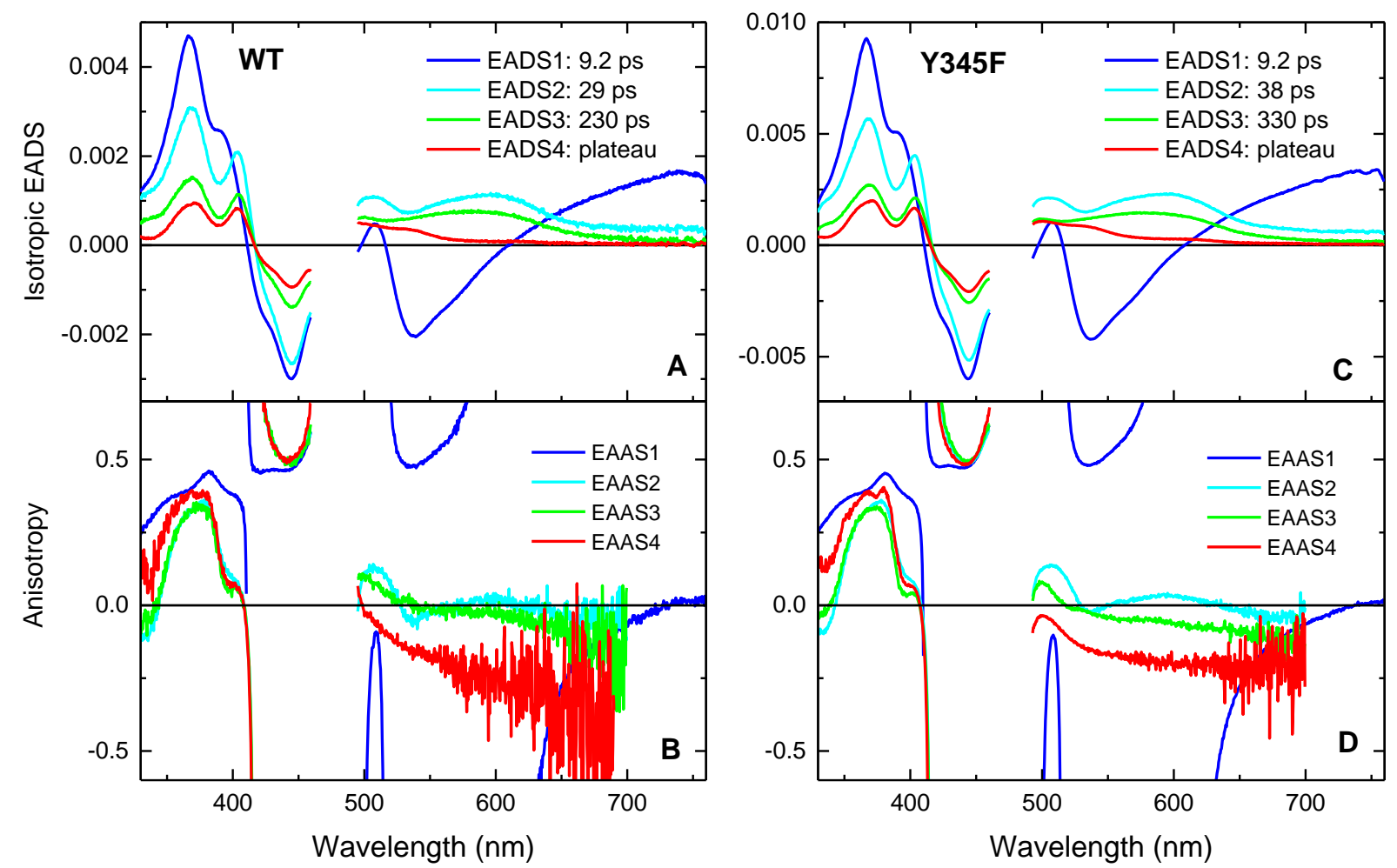

Figure 3. Top: Isotropic EADS deduced from the global analysis of the polarized transient absorption spectra of $M m$ CPDII in $\mathrm{H}_{2} \mathrm{O}$ buffer (A: WT; C: Y345F) with a sum of 3 exponentials and a plateau. Bottom: Corresponding EAAS (B: WT; D: Y345F). Exceedingly noisy parts of these spectra above $700 \mathrm{~nm}$ have been masked. 


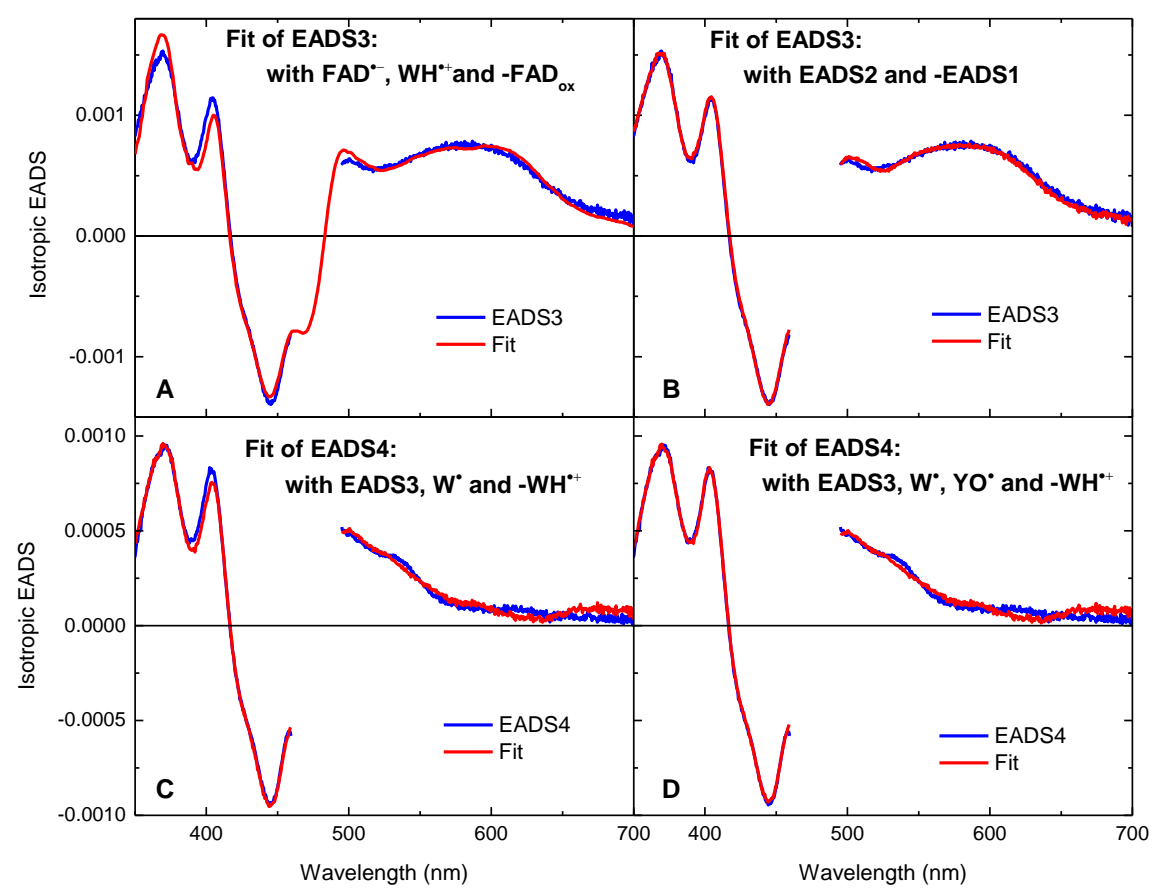

Figure 4. Spectral fittings of EADS3 (A, B) and EADS4 (C, D) of WT-MmCPDII with weighted sums of component spectra (see details in $§ 4.2$ and ESI-§4).

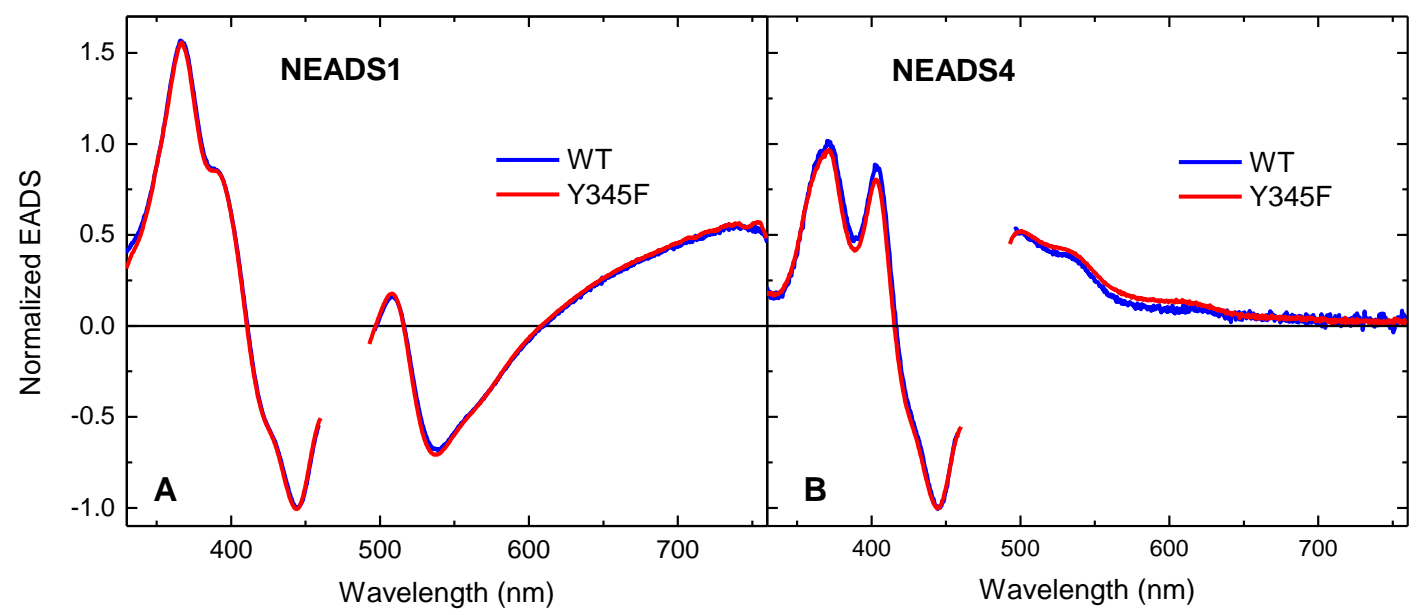

Figure 5. Comparison of the normalized EADS1 (A) and EADS4 (B) of WT-MmCPDII and its Y345F mutant. 


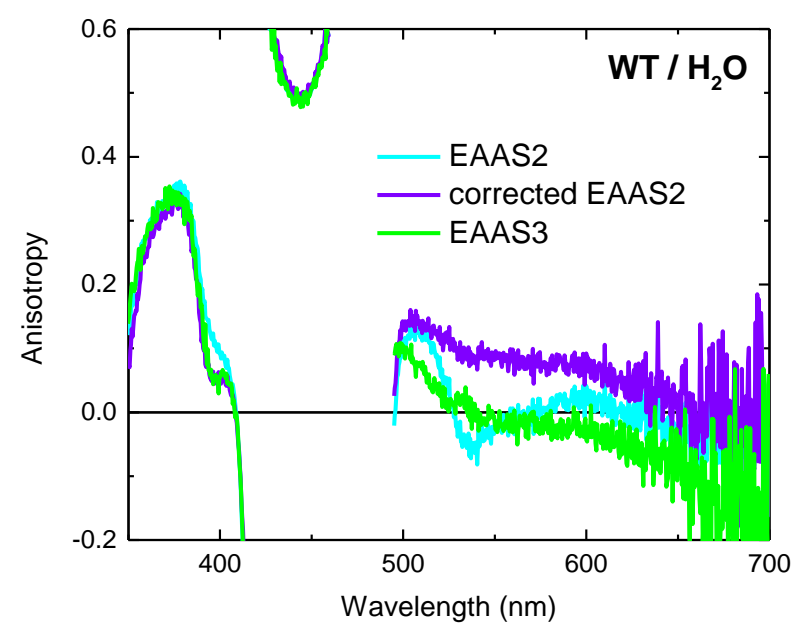

Figure 6. Corrected EAAS2 (purple) of WT- $M m$ CPDII in $\mathrm{H}_{2} \mathrm{O}$ buffer. The original EAAS2 (cyan) and EAAS3 (green) are given for reference.

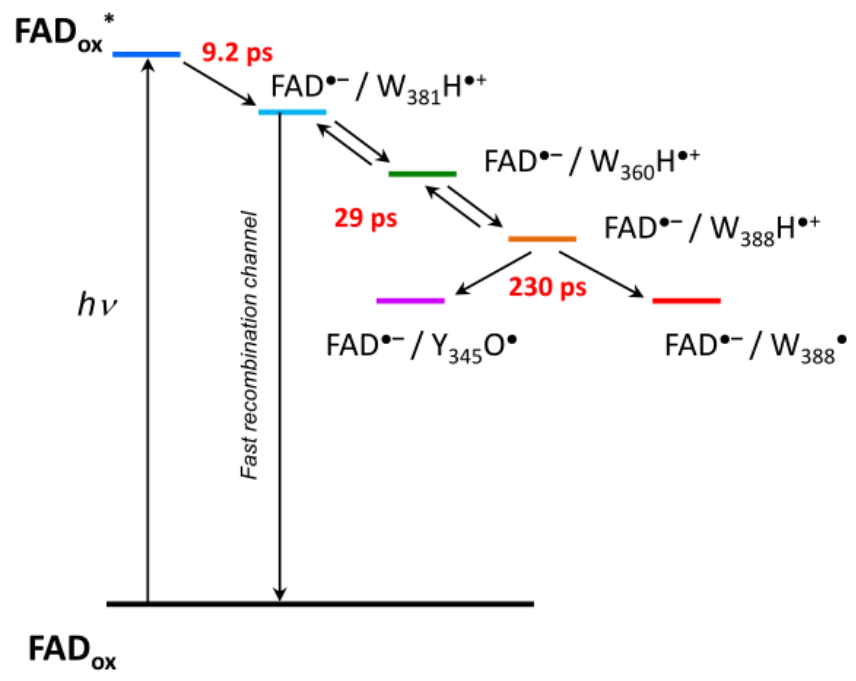

Figure 7. Reaction scheme for the primary photoreduction events in MmCPDII. 


\section{TABLES}

Table 1. Time constants of the global multiexponential fit of the polarized transient absorption spectra of $M m$ CPDII, WT in $\mathrm{H}_{2} \mathrm{O}$ and $\mathrm{D}_{2} \mathrm{O}$ buffer, and $\mathrm{Y} 345 \mathrm{~F}$ in $\mathrm{H}_{2} \mathrm{O}$ buffer. Fit errors $( \pm 2 \sigma)$ are indicated in smaller characters. The coefficient of determination $\left(\mathrm{R}^{2}\right)$ is given in the last column.

\begin{tabular}{llllll}
\hline protein & buffer & $\tau_{1}(\mathrm{ps})$ & $\tau_{2}(\mathrm{ps})$ & $\tau_{3}(\mathrm{ps})$ & $\mathrm{R}^{2}$ \\
\hline \multirow{2}{*}{$\mathrm{WT}$} & $\mathrm{H}_{2} \mathrm{O}$ & $9.2 \pm 0.5$ & $29 \pm 4$ & $230 \pm 40$ & 0.999042 \\
& $\mathrm{D}_{2} \mathrm{O}$ & $10.7 \pm 0.8$ & $50 \pm 8$ & $570 \pm 190$ & 0.997678 \\
\hline $\mathrm{Y} 345 \mathrm{~F}$ & $\mathrm{H}_{2} \mathrm{O}$ & $9.2 \pm 0.2$ & $38 \pm 2$ & $330 \pm 40$ & 0.999834 \\
\hline
\end{tabular}

Table 2. Evolution-associated anisotropies taken at $600 \mathrm{~nm}$ (averaged over $6 \mathrm{~nm}$ ), after initial decay of the excited state $\left(\mathrm{r}_{\mathrm{i}}=\right.$ EAASi $\left.(600 \mathrm{~nm})\right)$. Values of EAAS2 corrected from the excited $\mathrm{FAD}_{\mathrm{ox}}$ contribution $\left(\mathrm{r}_{2}{ }^{\prime}\right)$ are given in the last column.

\begin{tabular}{lllcc||c}
\hline protein & buffer & $\mathrm{r}_{2}$ & $\mathrm{r}_{3}$ & $\mathrm{r}_{4}$ & $\mathrm{r}_{2}{ }^{\prime}$ \\
\hline \multirow{2}{*}{$\mathrm{WT}$} & $\mathrm{H}_{2} \mathrm{O}$ & 0.023 & -0.029 & -0.26 & 0.073 \\
& $\mathrm{D}_{2} \mathrm{O}$ & 0.038 & -0.088 & -0.13 & 0.076 \\
\hline $\mathrm{Y} 345 \mathrm{~F}$ & $\mathrm{H}_{2} \mathrm{O}$ & 0.032 & -0.063 & -0.20 & 0.073 \\
\hline
\end{tabular}


Table 3. Expected anisotropies at $600 \mathrm{~nm}$ of the different $\mathrm{WH}^{\bullet+}$ radicals of the photoreduction site, according to the crystal structure of $M m \mathrm{CPDII}(\mathrm{PDB} 2 \mathrm{XRY}){ }^{7}$ Errors indicated in small characters correspond to variations of $\pm 5^{\circ}$ of the directions of both $\mathrm{FAD}_{\mathrm{ox}}$ and $\mathrm{WH}^{\bullet+}$ transition moments within the molecular plane. The bottom line proposes at tentative correction of these values, including a contribution from $\mathrm{FAD}^{\bullet-}$ (see text).

\begin{tabular}{cccc|c}
\hline & $\mathrm{W}_{381}$ & $\mathrm{~W}_{360}$ & $\mathrm{~W}_{388}$ & $\mathrm{~W}_{421}$ \\
\hline raw & $0.11 \pm 0.04$ & $0.20 \pm 0.06$ & $-0.19 \pm 0.01$ & $-0.04_{ \pm 0.03}$ \\
\hline corrected & $0.05 \pm 0.04$ & $0.12 \pm 0.06$ & $-0.19_{ \pm 0.01}$ & $-0.07 \pm 0.03$ \\
\hline
\end{tabular}

\section{ASSOCIATED CONTENT}

Electronic supplementary information (ESI) available: 1) Steady-state absorption spectra, 2) Transient absorption and anisotropy spectra, 3) Global kinetic analysis, 4) Spectral fitting.

\section{AUTHOR INFORMATION}

\section{Corresponding Authors}

*Pascal Plaza: Email: pascal.plaza@ens.fr. Phone: +33 144322414.

*Lars-Oliver Essen: Email: essen@,chemie.uni-marburg.de. Phone: +49 64212822032. 